\title{
ICTs in Speech and Language Therapy
}

\author{
http://dx.doi.org/10.3991/ijep.v4i1.3280 \\ Athanasios S. Drigas and Alexia Petrova \\ NCSR DEMOKRITOS, Institute of Informatics and Telecommunications, Net Media Lab, Athens, Greece
}

\begin{abstract}
Over the past decade speech and language therapy has taken an interesting turn towards the use of information communication technologies (ICTs) for diagnosis of disorders and delivery of therapy. In many cases ICTs have worked as assistive tools to therapists, while in others as sole providers of therapy, especially in remote areas. In this report we provide a brief overview of the most representative articles for applications and assistive technologies used for assessment and intervention purposes in Speech Therapy according to the type of disorders.
\end{abstract}

Index Terms-ICT, speech and language therapy, language disorders, speech disorders, assessment, intervention.

\section{INTRODUCTION}

Speech has always been the most natural and most frequently used mean of human communication with the role of fulfilling the information transmission between individuals [1]. In that direction, the speech and language therapy profession has expanded rapidly, especially over the past 30 years, with complex clinical education and practices, while recently making use of available Information and Communications Technologies (ICTs) [2].

ICT in speech therapy can be used as a real clinical tool for diagnosis and intervention and so far includes webbased content, simulations and microworlds, adaptive systems, intelligent tutoring systems, virtual reality, handheld and mobile devices, virtual learning environments as well as tele-health [3].

The literature refers to the positive impact of ICT usage in children and adults with deficient speech and language skills. Research on the ICT usage for assessment, support and rehabilitation of communication disorders have revealed positive results in disorders caused by neurological conditions, autism, hearing impairment and acquired speech and language disorders [4].

Through the use of ICTs, therapists are given the possibility to assist their own clinical competences along with assistive technology so as to provide their patients with the most integrated therapy plans, in a flexible and innovative way. Moreover, they can be used as measurement instruments to promote research on the field of Speech Therapy [3, 4].

In this report we make an overview of the most popular applications that are used for assessment and intervention purposes of speech and language disorders both in children and adults. For presenting the ICTs in Speech Therapy, we have created a map consisting of two main categories: assessment and intervention. The first one includes assessment tools for adults and for children, while the second one is divided in sections according to the type of disorders.

\section{ICTS IN ASSESSMENT PROCEDURES}

Early diagnosis of speech and language disorders, in either children or adults, is of prime importance for any Speech and Language Therapist and the key to good chances for comprehensive rehabilitation. Waiting for speech deficiencies to decline by themselves may be proven hazardous and could result in poor communication skills, as well as damaged psychology and self-esteem. As Speech Therapy can sometimes be time-consuming and laborious for both therapists and patients, intelligent diagnosis and therapy systems have been created as a way to enhance language therapy efficiency [5].

"Telelogos", which was introduced by [6], is an "easy to use" web-based tool that allows SLTs to evaluate and find the optimum treatment for each patient. The system offers an alternative approach to remote and technologyassisted SLT and evaluates children through speech therapy, as well as learning disabilities tests. Furthermore, it allows communication with paralyzed, speech-impaired patients, teaches them to write, evaluates phonological awareness and maturity, and can also be used as a communicator between two disabled children.

In 2006, reference [7] introduced a PC-based integrated aid tool for the assessment and analysis of voice disorders. This tele-diagnosis software package is called "Diagnosis of Voice Disorders (DoVd)" and integrates the techniques of endoscopy, perceptual evaluation of voice and acoustic analysis in two main applications. The first one "MedivozCaptura"- records the signals involved in speech production, while the second -"WPCVox"- analyses them, thus objectively measuring their quality. The software, specially designed for use on a $\mathrm{PC}$, proposes a large voice and endoscopic image database that could be shared and used for research purposes.

Reference [8] conducted a research on patients with aphasia to identify whether there would be a difference on the results between the standardized face-to-face language assessment and a real-time telerehabilitation via video conferencing. A total of 32 aphasic patients, were examined by two Speech and Language Pathologists under the two conditions, in order to determine the influence of severity of language impairment on the ability to assess language skills. Results suggested that, even though the severity of aphasia challenges the assessment via telerehabilitation, yet it does not have an impact on the accuracy of the assessment results. Some subtests may be more arduous via telerehabilitation, due to difficulties such as audio and video quality deterioration; however these could be eliminated with the use of higher bandwidths.

Ref. [9] presented the results of their research regarding the intelligibility of dysarthric speakers. Their paper describes the development of a model providing reliable phoneme intelligibility scores calculated from three dif- 
ferent types of intelligibility models, namely models using phonemic features, or phonological features or a combination of the both, which occurred from alignments between the speech and the typical phonetic transcription of what the speaker was meant to say. 10550 recordings of consonant- vowel-consonant words were produced by 51 control speakers and 160 speakers with all types of dysarthria, with severity ranging from mild to severe. The findings of the study were reliable and objective enough to be used in a clinical tool.

Ref. [10] experimented on extraction of speech characteristics from clients' speech, that were recorded over a telephone, in order to develop an early screening system for cognitive decline. Those speech characteristics were compared and have been found similar to those extracted from recordings made in controlled environment. Results have shown that remotely recorded speech characteristics can be, will little algorithmic changes, reliable enough to be used in monitoring the onset and intervening on cognitive decline of aging people.

Ref. [11] introduced an internet based Speech Pathology Diagnostic Expert System in Greek, named APLo, suitable for assessing the oral language abilities of children aged between four and seven. The development methodology of this system is modeled and represented by a hybrid approach that integrates symbolic rules with neural networks providing indicators of disorder classification. The tool evaluates the answers of the user and provides a list of weaknesses or disorders located. The SLP can use this feedback to plan therapy or learning tasks and support speech and language issues.

\section{ICTS IN INTERVENTION PROSEDURES}

Assistive software has also found great use on intervention in people with speech and language disabilities, neurological disorders - genetic or acquired-, hearing impairment as well as autism spectrum disorders. ICTs in intervention should be designed in accordance to the disability, in an easy and understandable manner for the user aiming at allowing speakers to achieve the highest speech, language and cognitive performances possible $[12,13]$.

\section{A. Autism Spectrum Disorders}

Ref. [14] examines the addition of an affective/emotional dimension in the computer-aided learning or instruction methods (CAL/CAI systems) for handling autism interventions. This attachment is aiming at enhancing and mediating the teacher-child educational process with various interaction forms that are in accordance with the level of disability of the autistic person. The systems gives the instructions in form of an affective avatar, or synthesized speech (in Greek), or written in the screen or a combination of all three. For better recognition, Makaton symbols are presented together with the real images. The avatar responds to the child's actions by expressing emotions appropriate to each situation, imitating those of the teachers in similar situations. The system also allows the recording of the learning process of each child for further statistical analysis.

Ref. [15] evaluates the implementation of ACALPA edutainment platform for autistic children. The platform uses an affective avatar, synthesized speech and multimedia content (videos, images and sounds) aiming at supporting and facilitating the teacher-child interaction. The platform uses more and less complex modules, according to the child's capacities, with each one of them focusing on a specific learning domain. The results of the evaluation indicate that the avatar actually advances the educational process.

Ref. [16] describes a novel design prototype for teaching children with ASD, from preschoolers to 4th grade students, facial expression recognition, called Facial Expression Wonderland (FEW). Training is succeeded through playing a computer game based on the film 'Alice in Wonderland' comprised of three different training levels, with voice instructions. In order to move to the next level, children have to clear all tasks of each level.

Reference [17], through a long-term study, reports on the benefits of using the TROCAS customized platform which is a message board designed to support visual, auditory and context information. In a very simple way, this platform allows autistic children to share opinions with others through the use of "Like" and "Dislike" buttons, view other children's opinions and exchange messages. Study results have shown significant and consistent changes in students' communication patterns.

In their article, [18] present the use of a virtual reality application in autism treatment using 3D virtual pink dolphins in a 3D dolphinarium. This learning activity system (LAS) enables children to deal with their learning and behavioral needs and works as an external stimulus where various distractions can be thrown into and distract the subject. Therapists teach children how to interact with the $3 \mathrm{D}$ dolphins with the use of simple hand gestures to prompt the dolphin to respond. After a while, through this reinforcement, the child with ASD progresses and acquires other gestures, learns to respond to dolphin sounds and even dialogues with the virtual dolphins, thus obtaining communication skills.

\section{B. Hearing Impairment}

Reference [19] describes an e-training tool designed to help hearing-impaired children learn and practice words in Thai language more correctly. The tool uses speech technologies, e.g. speech recognition and computer animation, to overcome the limitations of the traditional face-to-face speech therapy on three aspects: Teaching, Training, and Tracking.

Ref. [20] developed a software system for Persian hearing-impaired children. With the use of a creative, entertaining combination of multimedia material the system offers about 300 videos of different articulations, plus hand tracked schemes of 26 different tongue-positioning, which facilitate the interaction and synchronization of language learning activities conducted both at home and in speech therapy centers. The results of the preliminary tests on hearing-impaired children aged from 2 to 6 years, showed the successful integration of the designed system into the convenient method used by speech therapists in Iran.

\section{Language Disorders}

Language disorders, affect the expression and understanding of language as well as reading and writing. Traditional therapy and ICTs, therefore, aim at improving the patients' ability to communicate by enhancing the remaining language abilities and restore the affected ones as much as possible [21].

Ref. [22] investigated the benefits of remote speechtherapy by interviewing six aphasic patients after receiv- 
ing a 6 month remote computer-based word-retrieval therapy. Their research concluded in a positive response by both patients and therapists. Patients reported a sense of increased autonomy and therapists outlined improvement in language abilities and increase in self-esteem. On the other side, however, was the amount of time spent in practice, which was deprived from other activities.

Ref. [23] recruited seven participants with word-finding difficulties associated with aphasia due to CVA, in order to examine whether remote speech-therapy can be beneficial in word-retrieval and in therapist time. Therapy was delivered 3-6 times a week for 27 weeks from the clients' home-computer. The software included a wide range of therapy tasks and feedback. Comparison between pre and post-therapy language assessments and participant interviews presented intensive use of the system and improvement on word-retrieval, proving this mode of therapy to be acceptable and effective.

Project CIRCA (Computer Interactive Reminiscence and Conversation Aid) as described by [24] is a computerbased multimedia tool that uses interactive multimedia in order to stimulate long-term memory, thus prompting verbal and non-verbal communication in people with dementia. After testing in two subject groups -one consisting of people with dementia and one of caregivers- it has been concluded that it augments patients' conversational autonomy; it promotes a relaxed interaction atmosphere and a more naturalistic conversation experience by removing pressure from the caregivers to continually prompt and finally CIRCA prompted memories from a number of individuals that none of the caregivers had heard before.

Ref. [25] developed and reviewed a computer-assisted treatment for word retrieval deficits using Cued Naming exercises of MossTalk Words. Their therapy system is a hierarchical, multi-modality cueing protocol under clinician-guided and self-guided instruction. Their review showed that computer-assisted treatment can benefit not only clinical-setting therapy, but also one-to-one speech therapy and concluded that those benefits extend to chronic aphasic individuals with significant phonologically based deficits.

Ref. [26] makes an evaluation of a hybrid desktophandheld system developed to support individuals with aphasia. The system uses images and sound on a PC, which are then downloaded to a mobile device and can be used by patients to support communication outside the home.

Ref. [27] investigated the effects of the Multi-Mode Matching Exercises module of MossTalk Words for improving word comprehension and retrieval in individuals with aphasia. Five patients with semantic and phonologic anomia were trained via computer with MossTalk multimode matching exercises (spoken and written word/picture matching) paired with spoken rehearsal. One month after training it was evident that picture naming performance remained above baseline levels proving that computerized lexical training exercises may lead to increases in word comprehension and production.

Ref. [28] introduced an Online Multimedia Language Assistant for individuals with aphasia called W2ANE. It has two main components, OMLA, an online multimedia library and ViVA, an adaptive vocabulary scaffold. The difference between W2ANE and similar systems is that W2ANE offers more modalities of multimedia data in- cluding videos and non-speech audio. Users are provided with online applications for looking up unknown words, practicing pronunciation and accessing content, thus enabling flexibility and language rehabilitation.

Ref. [12] presents the MAS learning platform for children with disabilities and cerebral palsy. MAS platform is a flexible and scalable software platform that integrates technologies in order to enable users to use adaptive games, aiming at monitoring and developing social and cognitive skills of children with disabilities. Preliminary results of implementation presented good acceptance levels both by users and educators.

Ref. [29] undertakes the matter of "telerehabilitation", which adds to "telehealth" the delivery of direct treatment through the use of ICTs. With the use of a virtual desktop the client is in real-time contact with the therapist and work together using on-screen materials, such as scanned worksheets and documents, or computer applications. After six weeks of treatment, all clients having suffered a stroke presented improvement in their cognitive and communication skills equivalent to a similar face-to-face treatment.

Ref. [30] also examines the effectiveness of MossTalk Words in two patients with anomia. The study proved that this computer-naming treatment program helped both patients improve their language abilities, maintain some of the therapeutic gains on the trained items and show generalization to a sentence production task one month post treatment.

In ref. [31] 17 people between 31 to 70 years old with chronic non-fluent aphasia and apraxia of speech participated in a 9-weeks treatment program using a computerized conversational script training program called AphasiaScriptsTM. The aim of the study was to investigate the relationship between amount of treatment and improvement on conversational script performance in persons with chronic non-fluent aphasia, and second to investigate the influence of severity of language impairment on this relationship. The results showed that the amount of treatment had a significant correlation with improvement both in content and in rate, especially in people with more severe aphasia.

A year later [32] examined the use of MossTalk Words in a patient with semantic dementia (SD) in an attempt to reinstate lost words. A client suffering from SD was exposed to the same errorless learning approach administered via a computer-based treatment for anomia. Four months after therapy, the client showed improvement on naming and also maintenance and generalization of gains, in contrast to other patients with SD reported in the literature that have been treated with different methods.

Reference [33] worked with adults with aphasia and apraxia of speech in a mixed paper-digital interface prototype using multimodal digital pens. In their paper they discuss the utility of multimodal paper-digital interfaces to assist therapy, which is believed to be particularly promising for speech-language therapy. The Livescribe Pulse Smartpen is a novel kind of digital pen that captures and recognizes paper-based handwriting with audio recording and playback and also allows custom-made applications to be deployed directly on the pen. Besides of being costeffective, this technology is also considered beneficial for supporting therapy. 


\section{Speech Disorders}

Speech disorders are presented through misarticulations of sounds, voice quality problems, dysfluencies and phonological disorders, while sometimes there may be a combination of the above mentioned problems. In most cases ICTs may assist the load of therapy for therapists and clients [13, 34].

\section{1) Dysarthria and Dyspraxia}

Ref. [14] describes an EU-funded research project called "Ortho-Logo-Paedia" (OLP) which aims at becoming a novel technological aid for improving the life quality of people with speech disorders. This project integrates speech training with speech recognition technology making these facilities available over the Internet. The system has three major components: 1. OPTACIA that provides the client with real-time visual feedback about her/his speech 2. GRIFOS, which is a speaker-dependent, smallvocabulary, automatic speech recognition system and 3 . TELEMACHOS, which will apply distance-learning principles based on web database technology to provide the system's remote tutoring and monitoring ability.

Ref. [35] presents results of trials of the STARDUST (Speech Training and Recognition for Dysarthric Users of Assistive Technology) to evaluate how Automatic speech recognition (ASR) can be used in a computerized training package to help patients with severe dysarthria improve the recognition likelihood and consistency of their vocalizations. Initial results presented good speech recognition rates for people with even the most severe dysarthria.

Ref. [36] introduces the development of STRAPTk, as part of the STARDUST project, a speech training toolkit for severely dysarthric speakers that aims at providing such speakers with access to devices for communication. The users of STRAPTk operate the software with a console allowing the instructors and clients to work at a high level graphic environment. The software prompts the patient to speak and then receive visual feedback, and at the same time gives the therapist the opportunity to go over the client's performance by reviewing the recorded speech material. The results of the trials on 5 severely dysarthric speakers showed that 3 out of 5 users presented an increase in the mean recognition probabilities for most words.

Ref. [37] describes a computer program based on the Lee Silverman Voice Treatment (LSVT) for Parkinson disease patients aiming at improving the voice and speech of patients, while at the same time overcoming the barriers of traditional LSVT. The paper presents the research and development of the virtual LSVT and also detects the disadvantages of the project that mainly focus on the lack of clinical judgment regarding the client's performance.

Ref. [34] presents "SpeechKit", a multimedia system used to assist in the intervention on motor impaired patients. Therapists may use screen prompts together with high quality digitized speech, instead of the usual paperbased prompts, thus making their work more effortless. In order for the system to be developed, the Asymetrix Toolbook has been used, whilst currently there is a more advanced system under development, specifically aimed at people with motor speech disorders.

Ref. [21] presents the results of the use of three different algorithms -DIRECT, Powell, and Nelder-Mead optimization algorithms- on a new magnetic localization sys- tem that aims at tracking tongue motion in the 3-D oral cavity. A magnet permanently attached to the tongue is used as a tracer to send signals to a 3-D magnetoinductive sensor array outside the mouth and wirelessly transmit them to a computer. The Powell algorithm has been found to more sufficient for the intended application in real-time tracking of the tongue motion.

Ref. [38] describes "E-learning based Speech Therapy" (EST) a tool used in the Netherlands aiming at providing remote, personalized, yet cost-effective Speech Therapy to dysarthric patients. A central server hosts two types of audio files: target speech files in MP3 format and recorded speech files uploaded by patients in wav format, accessible by a desktop computer or a laptop with internet connection. Therapists provide their patients with a personalized speech training program from the audio examples of target speech, stored at the server that patients download, listen and imitate as accurate as possible. Finally, their utterances are compared to the examples and uploaded to the server. The drawback of this method is the lack of visual feedback that would enhance their auditory discrimination abilities, that may be affected by subsequent cognitive problems or hearing loss.

\section{2) Voice Disorders}

Ref. [39] investigated the telehealth model of delivering voice therapy at a distance in terms of feasibility, client satisfaction and effectiveness of therapy. Data was gathered to develop a telehealth vocal rehabilitation protocol, while 12 participants received face-to-face therapy and 19 were treated via video-teleconferencing. Results pointed that via telehealth, patients have received the same quality of therapy and revealed high satisfaction. However, successful operationalization was diminished by administrative and technical issues.

Ref. [40] presents one of 16 MedCal e-learning projects, especially created for training Medical or Speech Therapy students on impaired voice quality and hoarseness, called DYSPHONIA. The program contains voice samples and analysis along with images and video clips of various pathological conditions. It offers students the opportunity to witness examination techniques, therapeutic sessions, and surgical interventions.

\section{3) Articulatory and Phonological Disorders}

Ref. [41] describes the first web-based tool for technology-assisted speech therapy called "Telelogos" tested in Greece and the UK. The tool contains -amongst others- a series of tests that allow therapists to assess the phonological maturity of the child and analyze its phonological system and then decide on the best treatment for each client.

Ref. [42] presents "Pre-Lingua", "Vocaliza" and "Cu'entame", three speech therapy tools aiming at helping people improve their communication abilities in terms of phonology, articulation and descriptive and comprehensive language respectively. Even though they are focused on Spain and Latin America, they may supply an easy interface for speech therapy in any language, encouraging improvements on functionality and robustness of informatic applications.

TERAPERS project is a system that assists therapists when treating dyslalia in Romanian preschoolers as described by [43]. The system involves a wide range of research areas including artificial intelligence, virtual reali- 
ty, digital signal processing and digital electronic and psychology. The main advantage of this system is that the therapist can save time from creating exercises and can be more concentrated on therapy.

Ref. [44] introduced an e-learning system for improving articulation in Greek preschoolers. The software that could be downloaded from the internet, recreated a game named "You've got talent" coaching the child in multiple articulation tasks and also providing feedback. The results of the study on this software showed that children not only improved their articulation but they also increased on language activities success by acquiring new vocabulary.

Ref. [45] presents an improved CBST system that was designed, named LOGOMON (Logopedics Monitor), which contains Children Manager, 3D Articulator Model, and Homework Manager all installed on the child's PC aiming at improving pre-school children's articulation. The advantages of using an expert system to generate exercise were more therapy time, predictability, explanation of results and a close interpersonal relationship between the speech therapist and the child.

\section{CONCLUSION}

This current study summarized some of the most representative studies of the last decade regarding the ICT usage in Speech Therapy that could facilitate the life of patients with speech and language disorders, therapists and caregivers as well. Even though at first therapists were reluctant about the appearance of technology in their field of ascendency, it seems that their behavior has turned significantly over the past decade. ICT is now considered a tool that assists and sometimes even achieves quicker and more efficient results where traditional therapy fails. We attempted to present the multiple types of ICT in Speech therapy according to assessment, intervention and types of disorders. There are domains that are efficiently covered by the existing ICTs and there are others for whom research is still in an early stage and further needs to be conducted.

\section{REFERENCES}

[1] Németh, G., Olaszy, G., Vicsi, K., \& Fegyó, T. Talking machines?!-. Infocommunications Journal, 8. (2009)

[2] Hoben, K., \& Morris, J. PATSy: innovations in learning for speech and language therapy. Bulletin of the Royal College of Speech and Language Therapists, 2005.

[3] Cox, R. Technology-enhanced research: educational ICT systems as research instruments. Technology, Pedagogy and Education, 16(3), 337-356, 2007. http://dx.doi.org/10.1080/14759390 701614470

[4] Danubianu, M., Tobolcea, I., \& Pentiuc, S. G. Advanced Technology in Speech Disorder Therapy of Romanian Language. arXiv preprint arXiv:0912.3969, 2009.

[5] Popovici, D. V., \& Buică-Belciu, C. Professional challenges in computer-assisted speech therapy. Procedia-Social and Behavioral Sciences, 33, 518-522, 2012. http://dx.doi.org/10.1016/ j.sbspro.2012.01.175

[6] Glykas, M., \& Chytas, P. Next generation of methods and tools for team work based care in speech and language therapy. Telematics and Informatics, 22(3), 135-160, 2005. http://dx.doi.org/10.1016/ j.tele. 2004.04 .002

[7] Godino-Llorente, J. I., Sáenz-Lechón, N., Osma-Ruiz, V., Aguilera-Navarro, S., \& Gómez-Vilda, P. An integrated tool for the diagnosis of voice disorders. Medical engineering \& physics, 28(3), 276-289, 2006. http://dx.doi.org/10.1016/ j.medengphy.2005.04.014
[8] Hill, A. J., Theodoros, D. G., Russell, T. G., Ward, E. C., \& Wootton, R. The effects of aphasia severity on the ability to assess language disorders via telerehabilitation. Aphasiology, 23(5), 627642, 2009. http://dx.doi.org/10.1080/02687030801909659

[9] Van Nuffelen, G., Middag, C., De Bodt, M., \& Martens, J. P. Speech technology-based assessment of phoneme intelligibility in dysarthria. International journal of language \& communication disorders, 44(5), 716-730, 2009. http://dx.doi.org/10.1080/136828 20802342062

[10] Rapcan, V., D'Arcy, S., Penard, N., Robertson, I. H., \& Reilly, R. B. The Use of Telephone Speech Recordings for Assessment and Monitoring of Cognitive Function in Elderly People. In Proceedings of 10th Annual conference of the International Speech Communication Association (Interspeech 2009), Brighton (pp. 6-10), September 2009.

[11] Toki, E. I., Pange, J., \& Mikropoulos, T. A. An Online Expert System for Diagnostic Assessment Procedures on Young Children's Oral Speech and Language. Procedia Computer Science, 14, 428-437, 2012. http://dx.doi.org/10.1016/j.procs.2012.10.049

[12] Paniagua Martín, F., Colomo Palacios, R., \& García-Crespo, Á. MAS: learning support software platform for people with disabilities. In Proceedings of the 1st ACM SIGMM international workshop on Media studies and implementations that help improving access to disabled users (pp. 47-52). ACM, October 2009.

[13] Öster, A. M., House, D., Protopapas, A., \& Hatzis, A. Presentation of a new EU project for speech therapy: OLP (Ortho-LogoPaedia). In Proceedings of the $X V$ Swedish Phonetics Conference (Fonetik 2002) (pp. 29-31), May 2002.

[14] Luneski, A., Konstantinidis, E. I., Hitoglou-Antoniadou, M., \& Bamidis, P. D. Affective computer-aided learning for autistic children. In 1st Workshop of Child, Computer and Interaction (WOCCI '08). Chania, Greece, October 2008.

[15] Konstantinidis, E. I., Hitoglou-Antoniadou, M., Luneski, A. Bamidis, P. D., \& Nikolaidou, M. M. Using affective avatars and rich multimedia content for education of children with autism. In Proceedings of the 2nd International Conference on PErvasive Technologies Related to Assistive Environments (p. 58). ACM, June 2009. http://dx.doi.org/10.1145/1579114.1579172

[16] Tseng, R. Y., \& Do, E. Y. L. Facial expression wonderland (FEW): a novel design prototype of information and computer technology (ICT) for children with autism spectrum disorder (ASD). In Proceedings of the 1st ACM International Health Informatics Symposium (pp. 464-468). ACM, November 2010.

[17] da Silva, M. L., Simões, C., Gonçalves, D., Guerreiro, T., Silva, H., \& Botelho, F. TROCAS: communication skills development in children with autism spectrum disorders via ICT. In HumanComputer Interaction-INTERACT 2011 (pp. 644-647). Springer Berlin Heidelberg, 2011.

[18] Chia, N. K. H., Cai, Y., Kee, N. K. N., Thalmann, N., Yang, B., Zheng, J., \& Thalmann, D. Learning Activity System Design for Autistic Children Using Virtual Pink Dolphins. In $3 D$ Immersive and Interactive Learning (pp. 105-121). Springer Singapore, 2013.

[19] Witsawakiti, N., Suchato, A., \& Punyabukkana, P. Thai Language E-Training for the Hard of Hearing. Special Issue of the International Journal of the Computer, the Internet and Management, Vol. 14 No.SP1, August, 2006.

[20] Bastanfard, A., Rezaei, N. A., Mottaghizadeh, M., \& Fazel, M. A novel multimedia educational speech therapy system for hearing impaired children. In Advances in Multimedia Information Processing-PCM 2010 (pp. 705-715). Springer Berlin Heidelberg.

[21] Cheng, C., Huo, X., \& Ghovanloo, M. Towards a magnetic localization system for 3-D tracking of tongue movements in speechlanguage therapy. In Engineering in Medicine and Biology Society, 2009. EMBC 2009. Annual International Conference of the IEEE (pp. 563-566). IEEE, September 2009.

[22] Wade, J., Mortley, J., \& Enderby, P. Talk about IT: Views of people with aphasia and their partners on receiving remotely monitored computer-based word finding therapy. Aphasiology, 17(11), 1031-1056, 2003. http://dx.doi.org/10.1080/026870 30344000373

[23] Mortley, J., Wade, J., \& Enderby, P. Superhighway to promoting a client-therapist partnership? Using the Internet to deliver word-retrieval computer therapy, monitored remotely with mini- 
mal speech and language therapy input. Aphasiology, 18(3), 193211, 2004. http://dx.doi.org/10.1080/02687030344000553

[24] Gowans, G., Campbell, J., Alm, N., Dye, R., Astell, A., \& Ellis, M. Designing a multimedia conversation aid for reminiscence therapy in dementia care environments. In $\mathrm{CHI}^{\prime} 04$ Extended $\mathrm{Ab}$ stracts on Human Factors in Computing Systems (pp. 825-836). ACM, April 2004.

[25] Fink, R., Brecher, A., Sobel, P., \& Schwartz, M. Computerassisted treatment of word retrieval deficits in aphasia. Aphasiology, 19 (10-11), 943-954, 2005. http://dx.doi.org/10.1080/ 02687030544000155

[26] Boyd-Graber, J. L., Nikolova, S. S., Moffatt, K. A., Kin, K. C., Lee, J. Y., Mackey, L. W., ...\& Klawe, M. M. Participatory design with proxies: developing a desktop-PDA system to support people with aphasia. In Proceedings of the SIGCHI conference on Human Factors in computing systems (pp. 151-160). ACM, April 2006.

[27] Raymer, A. M., Kohen, F. P., \& Saffell, D. Computerised training for impairments of word comprehension and retrieval in aphasia. Aphasiology, 20(02-04), 257-268, 2006.

[28] Ma, X., Nikolova, S., \& Cook, P. R. W2ANE: when words are not enough: online multimedia language assistant for people with aphasia. In Proceedings of the 17th ACM international conference on Multimedia (pp. 749-752). ACM, October 2009.

[29] Brennan, D., Georgeadis, A., \& Baron, C. Telerehabilitation tools for the provision of remote speech-language treatment. Topics in Stroke Rehabilitation, 8(4), 71-78, 2002. http://dx.doi.org/10.1310/U7KV-DY7U-Q6QP-LVBP

[30] Jokel, R., Cupit, J., Rochon, E., \& Leonard, C. Relearning lost vocabulary in nonfluent progressive aphasia with MossTalk

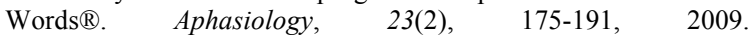
http://dx.doi.org/10.1080/02687030801943005

[31] Lee, J. B., Kaye, R. C., \& Cherney, L. R. Conversational script performance in adults with non-fluent aphasia: Treatment intensity and aphasia severity. Aphasiology, 23(7-8), 885-897, 2009. http://dx.doi.org/10.1080/02687030802669534

[32] Jokel, R., Rochon, E., \& Anderson, N. D. Errorless learning of computer-generated words in a patient with semantic dementia. Neuropsychological rehabilitation, 20(1), 16-41, 2010. http://dx.doi.org/10.1080/09602010902879859

[33] Piper, A. M., Weibel, N., \& Hollan, J. D. Introducing multimodal paper-digital interfaces for speech-language therapy. In Proceedings of the 12th international ACM SIGACCESS conference on Computers and accessibility (pp. 203-210). ACM, October 2010.

[34] Calder, D. SpeechKit: a multimedia speech tool. In Proceedings of the 10th International Conference on Information Integration and Web-based Applications \& Services (pp. 647-650). ACM, November 2008.

[35] Hawley, M., Enderby, P., Green, P., Brownsell, S., Hatzis, A., Parker, M. \& Palmer, R. STARDUST; Speech Training And Recognition for Dysarthric Users of Assistive Technology. In 7th European Conference for the Advancement of Assistive Technology (AAATE 2003) (pp. 959-963), August 2003.

[36] Hatzis, A., Green, P., Carmichael, J., Cunningham, S., Palmer, R., Parker, M., \& O’Neill, P. An integrated toolkit deploying speech technology for computer based speech training with application to dysarthric speakers. In Proceedings of the 8th European Conference on Speech Communication and Technology (Eurospeech'03) (pp. 2213-2216), September 2003
[37] Cole, R., Halpern, A., Ramig, L., Van Vuuren, S., Ngampatipatpong, N., \& Yan, J. A virtual speech therapist for individuals with Parkinson's Disease. Educational technology-saddle brook then englewood cliffs NJ-, 47(1), 51, 2007.

[38] Beijer, L., \& Rietveld, T. Potentials of Telehealth Devices for Speech Therapy in Parkinson's Disease, 2011.

[39] Mashima, P. A. The Use of Video-Teleconferencing to Deliver Voice Therapy At-A-Distance (Doctoral dissertation, University of Cincinnati), 2011.

[40] Åkerlund, L., Hulting, A. L., \& Petersson, G. DysphoniaIllustrating a Nationwide Initiative to Provide Students with High Quality e-Learning Resources. International Journal of Innovation in Science and Mathematics Education (formerly CAL-laborate International), 6(1), 2012.

[41] Glykas, M., \& Chytas, P. Technology assisted speech and language therapy. International Journal of Medical Informatics, 73(6), 529-541, 2004. http://dx.doi.org/10.1016/ j.ijmedinf.2004.03.005

[42] Rodríguez, W., Saz, O., Lleida, E., Vaquero, C., \& Escartín, A. Comunica-tools for speech and language therapy. In Workshop on Child, Computer and Interaction, Chania, Grecia, October 2008.

[43] Danubianu, M., Pentiuc, S. G., Schipor, O. A., Nestor, M., Ungureanu, I., \& Schipor, D. M. TERAPERS-Intelligent Solution for Personalized Therapy of Speech Disorders. International Journal On Advances in Life Sciences, 1(1), 26-35, 2009.

[44] Toki, E. I., \& Pange, J. E-learning activities for articulation in speech language therapy and learning for preschool children. Procedia-Social and Behavioral Sciences, 2(2), 4274-4278, 2010. http://dx.doi.org/10.1016/j.sbspro.2010.03.678

[45] Schipor, O. A., Pentiuc, S. G., \& Schipor, M. D. Improving computer based speech therapy using a fuzzy expert system. Computing and Informatics, 29(2), 303-318, 2012.

\section{AUTHORS}

Athanasios Drigas is a Senior Researcher at N.C.S.R. Demokritos. He is the Coordinator of Telecoms Lab and founder of Net Media Lab since 1996. From 1985 to 1999 he was the Operational manager of the Greek Academic network. He has been the Coordinator of Several International Projects, in the fields of ICTs, and e-services (elearning, e-psychology, e-government, e-inclusion, eculture etc). He has published more than 200 articles, 7 books, 25 educational CD-ROMs and several patents. He has been a member of several International committees for the design and coordination of Network and ICT activities and of international conferences and journals. (e-mail: dr@iit.demokritos.gr).

Alexia Petrova is a Speech and Language Therapist. She is also a researcher and has participated in research projects regarding Cognitive Science at N.C.S.R. Demokritos. (e-mail: alexiapetrova@yahoo.gr).

Submitted 23 October 2013. Published as re-submitted by the authors 23 January 2014. 